https://doi.org/10.15407/ujpe65.8.735

\title{
TO THE 90th BIRTHDAY OF PETRO IVANOVYCH FOMIN (1930-2011)
}

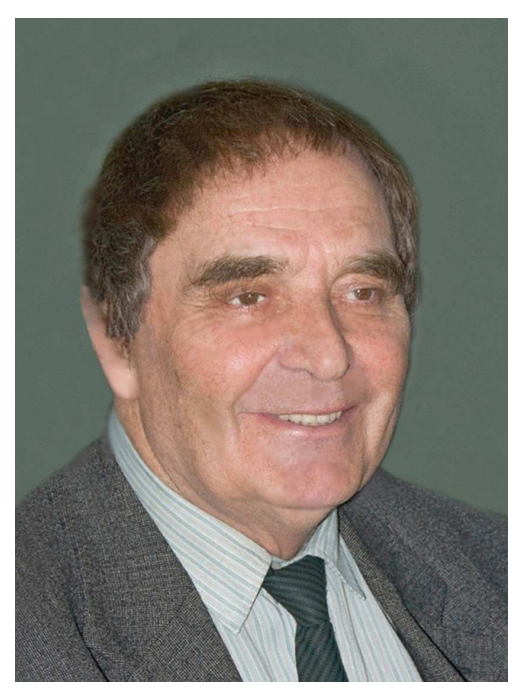

June 20, 2020 marked the 90th anniversary of the birth of the outstanding physicist-theorist Petro Ivanovych Fomin, Corresponding Member of the National Academy of Sciences of Ukraine (NASU), Honored Worker of Science and Technology of Ukraine, Laureate of the State Prize of Ukraine in science and tecnnology, Laureate of the M.P. Barabashov Prize and the D.V. Volkov Prize of the NASU, Professor, Dr. Sci. in physics and mathematics.

Petro Ivanovych was born on June 20, 1930 in the village of Zhykharevo (Orel region, Russia). After successfully graduating from the Kharkiv State University in 1953, he entered the post-graduate course of this university, where he worked under the scientific supervision of the world-famous physicisttheorist Academician Aleksandr Il'ich Akhiezer.

Since 1957, P.I. Fomin had been working at the Kharkiv Institute of Physics and Technology, where he defended his $\mathrm{PhD}$ and doctoral dissertations.
Since 1972, his creative destiny had been associated with the M.M. Bogolyubov Institute for Theoretical Physics of the NASU (Kyiv), where P.I. Fomin became a head of the Department of astrophysics and elementary particles and, later, a Chief Scientist.

In 1990, P.I. Fomin was elected a Corresponding Member of the NASU. He is an author of well-known fundamental works in the quantum field theory, the theory of elementary particles, astrophysics, and cosmology. In those works, he put forward a number of profound physical ideas and obtained a number of remarkable results. The works by P.I. Fomin on elementary particle physics and quantum field theory are devoted to the study of the structure of the physical vacuum and the manifestations of its properties in various physical processes. He was the first who pointed out and considered the mechanism of dynamic mass formation for quarks and hadrons, which is associated with the formation of the quark-antiquark vacuum condensate owing to a relativistic analog of the Cooper pairing in the strong (supercritical) quarkgluon interaction mode. This approach and the relevant conclusions gained a wide resonance in the world literature.

In 1973, P.I. Fomin showed, for the first time in the literature, that the unification of the principles of general relativity and quantum field theory brings about the phenomenon of the gravitational instability of vacuum. This phenomenon provides a basic possibility for the spontaneous quantum birth of a spatially closed universe. The corresponding theory, being supplemented with the cosmological "inflation" concept, allows the fundamental issues concerning the origin of our universe to be solved and sheds light on the nature of the so-called Big Bang. The quantum-field approach to the cosmological problem initiated by 
P.I. Fomin started a new direction, quantum cosmology, which is now rapidly developing. In the theory of gravitation, P.I. Fomin studied a number of physical effects associated with the existence of the event horizon. The axisymmetric solution obtained by him for the equations of the general theory of relativity adequately replenished the set of classical solutions in this domain. In his works, P.I. Fomin also developed the theory of gravitational quantization of the space-time on Planck scales, which resulted in the appearance of new concepts concerning a physical mechanism that allows one to avoid divergences in the quantum field theory.

In the domain of astrophysics, P.I. Fomin proposed an original solution to the problem dealing with the physical nature of the high-energy activity of quasars, radio galaxies, and nuclei of active galaxies. Such an activity manifests itself, in particular, in the form of relativistic jets.

In a series of works performed jointly by P.I. Fomin and the scientists from the Main Astronomical Observatory of the NASU, the high-energy gas-dynamic effects in astrophysical systems were studied. In particular, the explanation was proposed to the ringshaped waves of star formation, which is observed in a number of galaxies. Those waves propagate from the corresponding center owing to a train of powerful shock waves that are generated by the explosive activity of galactic nuclei and affect the interstellar environment. P.I. Fomin together with V.M. Mal'nev and A.P. Fomina explained the phenomenon of superpower radiation emitted by the Jupiter-Io system, which remained mysterious for a long time. Some in- teresting works by P.I. Fomin, which attracted the attention of geophysicists, were devoted to the solution of two long-standing problems concerning Earth's internal dynamics: the geomagnetic field inversion and the substantiation of the so-called macrodrop mode of the mantle convection.

P.I. Fomin was an author of more than 170 scientific papers. His work in quantum cosmology, relativistic astrophysics, and particle theory made an important contribution to the development of those branches of science.

Among his disciples, there are six doctors of science and 16 PhD's, who work fruitfully both in Ukraine and abroad. Petro Ivanovych was elected the President of the Ukrainian Society of Gravitation and the Editor-in-Chief of the journal Visnyk Astronomichnoi Shkoly (Bulletin of Astronomical School).

The talent of physicist-theorist, the originality and depth of thinking, great creative inspiration, optimism, and high human qualities gained Petro Ivanovych an authority and a respect among his colleagues and disciples. The blessed memory of Peter Ivanovich remains forever in the hearts of those who knew him and communicated with this outstanding scientist, a person with a good fortune and a sincere soul. 